\title{
SUPPORTING MULTICAST IN AD-HOC NETWORKS IN A HOTSPOT CONTEXT
}

\author{
Andreas Kassler ${ }^{1}$, Susana Sargento ${ }^{2}$, Adel Ben Mnaouer ${ }^{3}$, Chen Lei ${ }^{3}$, Pedro \\ Neves $^{2}$, Rui L. Aguiar ${ }^{2}$, Pedro M. Ruiz ${ }^{4}$ \\ 'Karlstad University, Sweden; ${ }^{2}$ Instituto de Telecomunicações, Universidade de Aveiro, \\ Portugal; ${ }^{3}$ Nanyang Technological University, Singapore; ${ }^{4}$ University of Murcia, Spain
}

\begin{abstract}
This paper discusses the usage of Ad-Hoc technologies as "hotspot extension" mechanisms. We propose a hybrid network where 802.16 links are used for providing high-bandwidth access, and local distribution is performed by AdHoc network nodes, thus covering arbitrary areas around the 802.16 stations, which might be connected to the Internet. We evaluate the performance of the system and its efficiency in providing QoS in multicast connections. We propose two extensions of the ODMRP and MAODV routing algorithms by augmenting them with a zone routing behavior, thus producing two new hybrid multicast routing algorithms: ZODMRP and ZMAODV. The simulation results show that ZODMRP provides the highest packet delivery ratio and lowest delay without introducing large overhead.
\end{abstract}

Key words: Multicasting; Hybrid MANET; Internet Connectivity; Hotspots.

\section{INTRODUCTION}

Ad-Hoc networks have gone through large developments over the last years. Researchers have investigated aspects of routing, multicasting, QoS and security in these networks. Currently, Ad-Hoc is reaching a stage where they can support the mixing of different services in order to provide an infrastructure useful for the common user. One such service is the basic network connection. Future users will be permanently connected, not only to their local environment, but also to the overall Internet. The "hotspot" concept is a clear indication of this trend, with more and more access points 
available for public usage. With the increased desire to be always connected, this concept will be certainly enlarged to an "extended hotspot" concept. The idea is to increase the range of hotspots through the automatic creation of Ad-Hoc networks based on connections to nodes increasingly nearer the hotspot range. We propose such a hybrid network where 802.16 links [1] [2] are used for providing high-bandwidth access, and local distribution is performed by multi-hop Ad-Hoc networks using IEEE 802.11. This allows for the covering of arbitrary areas around the 802.16 stations. Recent announcements of intrinsic laptop support for both 802.11 and 802.16 technologies [2], and the proposals of incentive-based charging schemes for Ad-Hoc networks [3], make this scenario quite feasible in the near future.

The paper discusses this scenario and analyzes multicast services on this environment. We discuss the usage of MAODV (Multicast Ad-Hoc Ondemand Distance Vector protocol) and ODMRP (On-Demand Multicast Routing Protocol) and variants to provide multicast support. The smooth integration of these different protocols and proposed evolutions will be presented. Furthermore, the adequate mapping of multicast requirements in the distribution 802.16 technology will also be discussed.

This paper is organized as follows. In section 2 the proposed overall network architecture is presented. Section 3 addresses the multicast mapping into 802.16 technologies. Multicast proposals in Ad-Hoc networks and their integration are described in section 4 . Simulation results are presented in Section 5. Finally, Section 6 reports the main conclusions.

\section{NETWORK ARCHITECTURE}

We propose to use 802.16 as an extension of access networks, providing high bandwidth access between the terminals and wired access network, and then extend this access by the creation of Ad-Hoc networks over 802.11. This situation can appear either with operator-owned hotspots or with dynamic situations, where 802.16/11-enabled users act as the gateway between the telecommunications operator and the other users (see Figure 1). The access router can be directly connected to the 802.16 base station (BS). As a broadband wireless access technology, 802.16 is intended to be used as a bridging solution between the access network and the backhaul wireless access technologies. Each one of these 802.11 Ad-Hoc networks is connected to the 802.16 technology through the (eventually fixed) subscriber station (SS) units. Each Ad-Hoc node is connected to the access point through a multi-hop path composed by mobile nodes in the Ad-Hoc network. 
In the uplink direction, the subscriber stations must forward the traffic to the base station in an on-demand basis, depending on the used uplink scheduling service. The mesh topology allows subscriber stations to communicate directly between them, avoiding forwarding all of its traffic to the base station. The purpose of this network is to be able to deliver and support any type of services and applications, as audio and video conferencing and streaming, to the end users, located in any hotspot network connected to the Internet through broadband wireless. Since the access network is composed by wired and fixed network, 802.16 network and wireless Ad-Hoc network, these networks need to be closely integrated.

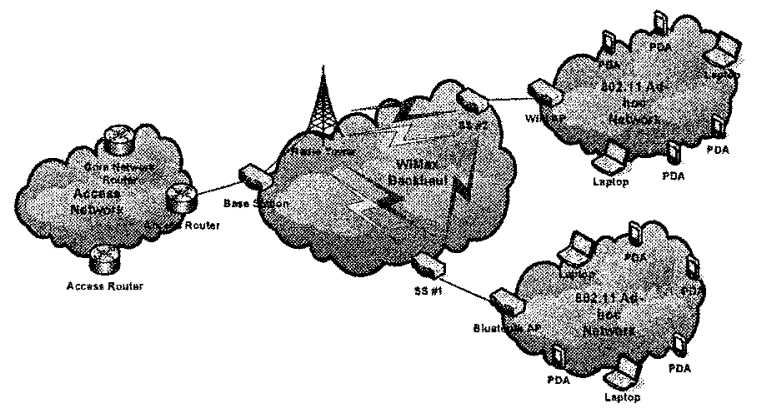

Figure 1. Network Architecture

\section{IEEE 802.16 MULTICAST}

While in 802.16 networks broadcast management and data connections are supported, there is no explicit multicast data transport connection defined. Instead, a range of connections is defined for multicast polling. These connections are used for the SSs to join multicast polling groups. Concerning multicast data transport connections, although there is no dedicated connection, it is possible to have a multicast connection for data.

The process for setting up a multicast connection is as follows. The BS should have information on the mobile nodes joining a multicast group. This can be provided by e.g. the access router which is then responsible to setup a multicast group inside the 802.16 network. The BS starts to create and associate a unicast transport connection with a specific SS. Then, this same connection is associated with all the SSs that belong to the multicast group. With this process, when multicast traffic is sent on this connection, all the SSs that belong to the multicast group are able to listen to this traffic. Inside Ad-Hoc networks, Ad-Hoc multicast routing protocols, as MAODV or 
ODMRP, are used to manage the multicast groups. An 802.16 multicast connection is only required if the multicast nodes belong to different SSs.

\section{AD-HOC MULTICAST INTEGRATION}

In multicast communications a source is sending only one packet with a group address as a destination. The network will be in charge of replicating that packet only when necessary to make it reach all destinations. The classical IP multicast protocols used in the Internet consists of the Internet Group Management Protocol (IGMP) [4] for group membership (or its IPv6 variant MLD) in combination with an IP based multicast routing protocol like Protocol Independent Multicast - Sparse Mode (PIM-SM) [5]. Because of Ad-Hoc network specific problems like frequent topology changes, unreliable links, battery constraints and limited capacity of mobile nodes, standard internet multicast routing protocols, introducing high overhead in maintaining efficient multicast delivery structures, do not perform well regarding scalability and performance in Ad-Hoc networks, especially when trees need to be re-organized frequently due to mobility. The benefits of multicast include the lower bandwidth consumption and increased scalability which makes support of multicast essential in Ad-Hoc networks.

\subsection{Multicasting in Ad-Hoc Environments}

Specific multicast routing protocols have been designed for Ad-Hoc networks, which can be classified according to the placement of the multicast delivery structure. In addition to pure flooding, which leads to a very high overhead in Ad-Hoc networks, we can distinguish between tree, mesh and location based multicast routing mechanisms. AMRoute [6], AMRIS [7], or MAODV [8] delivery structure is tree based. ODMRP [9] and CAMP [10] are mesh based. The motivation for mesh based approaches is due to the problems with tree-based approaches in the presence of mobile nodes because tree-structures are fragile and need to be frequently readjusted when connectivity changes. Using delivery meshes that span all multicast group members, multiple links do exist which provides redundancy to route breaks caused by mobility of nodes. In location-based multicasting (e.g. [11]), location information is used to define group membership and distribute multicast traffic.

Traditional (multicast) routing protocols for Ad-Hoc networks have not been designed to interoperate with fixed networks due to several reasons: The addressing architecture is different in Ad-Hoc networks, in which host- 
based routes are commonly used and addressing structure is flat compared to a hierarchical structure in the internet. In the Internet, two nodes sharing the same network part of their IP addresses are assumed to be in the same link, which is not necessarily the case in multi-hop networks. Classical internet multicast protocols offer superior performance in fixed networks than pure Ad-Hoc solutions, which are designed for frequent topology change. Due to these reasons, it is hard to design a multicast protocol that works efficiently in the Ad-Hoc part while still being able to interoperate with the fixed network. Therefore, it is desirable to design inter-working between Ad-Hoc and internet multicast protocols to achieve the best performance. Very little efforts, if any, have been started to provide multicasting in such hybrid AdHoc networks, see e.g. [12], [13], and those approaches present severe limitations in terms of functionality and generality.

\subsection{Integration of MANET with the public Internet}

In our scenario, where Ad-Hoc networks are connected through 802.16 with the internet, it is required to interoperate with a fixed IP network and support standard-IP multicast sources or receivers, and then, several extensions are required. These extensions must be designed in such a way that they are compatible with the standard IP Multicast mode, and they must allow standard IP nodes to take part in multicast communications without requiring any change. Therefore, an Ad-Hoc multicast routing protocol should support IGMP as a means to interoperate both with access gateways and standard IP nodes. In addition, the 802.16 BS must be enabled to setup a multicast group inside the 802.16 network, which requires information about what members of which multicast group are attached to what 802.16 SS.

We propose that all nodes in the Ad-Hoc fringe situated just one hop away from the gateway (denoted as Multicast Internet Gateways - MIG - in the Multicast MANET Routing Protocol - MMARP - [14] terminology) notify the access routers about the group memberships within the Ad-Hoc fringe. Any node within the Ad-Hoc fringe may become a MIG at any time if that node receives IGMP reports from multicast routers in the access network, because IGMP messages are sent with $T T L=1$. This requires in our architecture that the 802.16 network does not change the TTL of IGMP messages. This mechanism allows the multicast Ad-Hoc routing protocol to work with any IP multicast routing protocol in the access network and, therefore, it shields the multicast Ad-Hoc routing protocol operation from the protocols performing the intra-domain or inter-domain multicast routing. Therefore, MIGs must periodically advertise themselves to all other Ad-Hoc nodes as default multicast gateway to the fixed network. Ad-Hoc nodes can determine if they have to advertise themselves as MIG by receiving IGMP 
queries from the access router attached to the 802.16 BS. Such advertisement messages broadcasted from MIGs inform intermediate Ad-Hoc nodes about the path towards multicast sources in the access network, and thus the global internet. When such an advertisement reaches a receiver or neighbor of a receiver of a multicast group within the Ad-Hoc part, this node has to initiate a joining process using the multicast Ad-Hoc routing protocol towards the MIG. Once the MIG receives the request from an Ad-Hoc node to join, it sends an IGMP Report towards the access router thus updating group membership information. This ensures that IP multicast data from sources in the fixed network reach the destinations within the Ad-Hoc network. Also, the access router then notifies the 802.16 BS, which then sets up the multicast group within the 802.16 network to forward traffic to the correct 802.16 SS and thus to the proper Ad-Hoc island.

If an Ad-Hoc node is becoming a multicast source, it triggers the creation of a multicast distribution structure (tree or mesh) depending on the routing protocol in use. If the MIGs become aware of new multicast sources in the Ad-Hoc island, they notify the access routers about this information by sending IGMP reports towards the access router.

\subsection{Design of new Multicast Routing Protocols}

When all MIGs join multicast sources located in the MANET, this will result in high overhead. Therefore, we have to limit the control and data overhead of routing protocols. Combining the Zone Routing with reactive protocol features to form new hybrid protocols is expected to achieve good performance. Also, it has already been shown in [8] and [9] that MAODV and ODMRP present good performance. Therefore, our objective is to extend MAODV and ODMRP with a proactive behaviour.

The new hybrid protocol contains reactive and proactive (based on zone routing) elements. In the proactive mode, each node in the Ad-Hoc part of the network constructs and maintains a zone around it with a pre-configured zone radius. Each node thus maintains a zone routing table (ZRT) to record the multicast information of the nodes in its zone by periodically broadcasting an update packet. The time-to-live (TTL) value of the packet is set to the pre-configured zone radius. Each update packet includes multicast information of the source node (the node which is sending the update packet) to distribute information about the source node sending the update packet to members within its zone. In this way, any node receiving such an update packet knows immediately to which multicast groups a specific source node belongs. If such neighbours receive join requests for a specific multicast group, they can immediately determine to which source node to forward 
such a request. For MAODV, the update packet includes multicast group addresses that the source node belongs to, multicast group leader address, and the multicast group sequence number. For ODMRP, it includes multicast group addresses that the source node belongs to. When an Ad-Hoc node within the zone receives the update packet, it records all information in that packet, keeping the ZRT up-to-date by periodic broadcast. Also, we modify the way MAODV and ODMRP send out a join request. The resulting two protocol variants are described as ZMAODV and ZODMRP, respectively.

Sending RREQ in $Z M A O D V$ : When a node needs to send a RREQ, it first looks up the zone routing table to see whether there are nodes in the zone that belong to the multicast group the node intends to join. If there are, it compares other information, for example, multicast group sequence number and multicast group leader address, to ensure that the information recorded in the zone routing table is fresh. If there are still some nodes in the zone, the source node unicasts a RREQ to the nearest node and waits for the reply. If there are no nodes, the source broadcasts a RREQ (with TTL = zone radius). Only border nodes will handle the RREQ and all other nodes in the zone propagate the RREQ to border nodes. When a border node receives RREQ, it looks up its ZRT and continues the path finding procedure until some nodes belonging to multicast group receive the RREQ. That node will then generate a RREP and send it to the source node following the reverse path.

Sending Join Query in ZODMRP: The procedure sending Join Query in ZODMRP is similar to the procedure of sending RREQ in ZMAODV. The difference is when a node finds other nodes in its zone belonging to the multicast group. In this situation it does not only unicasts the Join Query to the node with the shortest path, but to all nodes belonging to the multicast group. This helps to build up the forwarding group as a mesh.

\section{EVALUATION OF ZMAODV AND ZODMRP}

We used GloMoSim [15] to compare the performance of ZMAODV and ZODMRP with MAODV and ODMRP. We distribute in total 50 Ad-Hoc nodes running 802.11 within an area of $1000 \times 1000 \mathrm{~m}$. Radio propagation range for each node is set to $250 \mathrm{~m}$ and channel capacity is $2 \mathrm{Mbps}$. Each simulation is executed for 500 s of simulation time, which corresponds to a typical time required for video news consumption within a hotspot. We assume the MANET to be the bottleneck and not the 802.16 backhaul network. We use constant bitrate UDP sources emulating typical audio and video streams. For audio, we use 240 byte packets, sent out every $30 \mathrm{~ms}$ at a total bitrate of $64 \mathrm{kbps}$. For video, we use 1500 byte packets, sent out every $80 \mathrm{~ms}$ at a total bitrate of $150 \mathrm{kbps}$. We select one node (mobility=0 $0 \mathrm{~m} / \mathrm{s}$ ) to 
be the access point towards the 802.16 access network. This node receives traffic from three (two audio and one video) other multicast sources and forwards it to the 802.16 access network. In addition, this node also multicasts two audio and one video stream that it receives from the 802.16 network towards the Ad-Hoc nodes. We use the following performance metrics to compare the performance of the multicast protocols: packet delivery ratio, which determines the effectiveness of the protocol (for interactive audio, packet delivery ratio must approach one for high quality experience), average packet delay and protocol overhead. Each node moves constantly with a randomly generated speed and moving directions are randomly selected. Nodes reaching the simulation boundary bounce back and continue to move. Although there are other mobility models available (like e.g. group mobility model), the random mobility model was selected as it stresses the routing protocols. Two different scenario setups are used. In the first setup we are interested in the protocol performance depending on the multicast group size. Here, we fixed the mobility of the nodes at $5 \mathrm{~m} / \mathrm{s}$ and vary the group size between 5 and 25 members in steps of 5 . In the second scenario, we fixed the multicast group size to 10 and vary the mobility of the nodes from 0 to $30 \mathrm{~m} / \mathrm{s}$ in steps of $5 \mathrm{~m} / \mathrm{s}$.

Figure 2 shows packet delivery ratio as a function of terminal speed. ODMRP shows good performance compared to MAODV even under high mobility. This is due to the mesh topology: the chances of packet delivery are high even if primary route is no longer available. ZODMRP outperforms ODMRP and ZMAODV performs better than MAODV, as the proactive zone feature helps to recover broken links. This is mostly visible at high mobility for ZMAODV. More packets are dropped in the direction towards the 802.16 network (denoted as at AP) as traffic is concentrated around the AP which leads to high collision ratio. Here, also the Z-variant outperforms the standard protocols and performance improvement is best for the ZMAODV when compared to MAODV at high mobility.

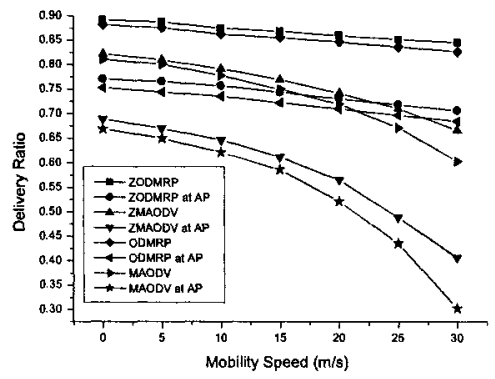

Figure 2. Delivery Ratio (mobility speed)

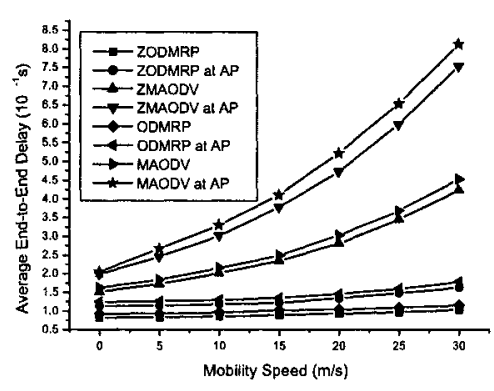

Figure 3. Average Delay (mobility speed) 
Figure 3 shows the average packet delay as a function of terminal speed. This is important in order to evaluate the suitability with respect to QoS provisioning. Again, ZODMRP outperforms ODMRP, which is better than ZAODV and AODV. Here, the performance degradation at high mobility is severe for MAODV ( $150 \mathrm{~ms}$ at $0 \mathrm{~m} / \mathrm{s}$ compared to $420 \mathrm{~ms}$ at $30 \mathrm{~m} / \mathrm{s})$ and ZMAODV. However, the delay for ZODMRP is nearly constant and below $100 \mathrm{~ms}$ even at high mobility, due to the intrinsic back-up routes provided by the mesh. Again, performance is significantly lower in direction towards the access point for all protocol variants.

Regarding protocol overhead, we have analyzed the number of data packets transmitted per number of data packets delivered to the destination, as a function of terminal speed. Both ZODMRP and ODMRP have highest number of transmissions (around 3 data packets transmitted per received data packet) due to the mesh structure exploiting multiple redundant routes. MAODV has higher overhead (around 2.3 packets) compared to 2.2 packets), especially for mobility higher than $15 \mathrm{~m} / \mathrm{s}$ than ZMAODV.

Concluding the analysis, we consider that the variants including the zone routing features are superior in providing QoS to the standard multicast routing protocols, when connecting Ad-Hoc islands to infrastructure based networks and thus to the internet. The performance increase is especially visible when comparing ZMAODV with MAODV. However, when considering QoS provisioning under the aspect of terminal mobility, ZODMRP is the method of choice.

\section{CONCLUSION}

We have presented a proposal for the integration of Ad-Hoc islands with infrastructure-based networks for providing multicast operations for users across both domains. The proposed scheme has interesting applications in hotspots' design and extensions thereof. This approach enables a seamless integration of wireless MANs and LANs with the public Internet, providing a common multicast framework. We have proposed and evaluated two new hybrid protocols ZODMRP and ZMAODV (based on the ODMRP and MAODV protocols) that were built using the zone routing concept. Extensive simulations of these protocols, and their original counterparts, revealed the superiority of the ZODMRP in providing predictable QoS guarantees for multicast sessions spanning over dissimilar and interconnected networks, without imposing higher overhead.

Our future work concentrates on exploiting Ad-Hoc mechanisms inside the 802.16 network, especially in the case of mobile and meshed 802.16 networks and evaluating the overhead of the announcement of default routes 
for the Ad-Hoc nodes. With respect to multicast routing in Ad-Hoc networks connected to the internet, we are working towards more realistic mobility models applicable to public hotspot environments. We will also consider other performance metrics like the connectivity of clients and delay for a user to connect to multicast sources located in the internet or Ad-Hoc fringe.

\section{ACKNOWLEDGEMENT}

The work described in this paper is partly based on results of IST FP6 Integrated Project DAIDALOS and "Ramon y Cajal" workprogramme.

\section{REFERENCES}

[1] IEEE 802.16-REVd/D2-2003, "IEEE Draft for Local and Metropolitan Area Networks Part 16: Air Interface for Fixed Broadband Wireless Access Systems", November 2003.

[2] Carl Eklund, Roger B. Marks, Kenneth L. Stanwood and Stanley Wang, "IEEE Standard 802.16: A Technical Overview of the Wireless MAN Air Interface for Broadband Wireless Access", IEEE Communications Magazine, June 2002.

[3] B. Lamparter, et al, "Charging Support for Ad Hoc Stub Networks", In Elsevier Journal of Comp. Com. Special Issue on Internet Pricing and Charging, Elsevier Science, Aug. 2003.

[4] Fenner, W., Internet Group Management Protocol, Version 2. RFC 2236, 11/ 1997.

[5] Estrin, D. et. al., "Protocol Independent Multicast Sparse Mode (PIM-SM): Protocol Specification." RFC 2362, June 1998.

[6]E. Bommaiah, M. Liu, A. McAuley, R. Talpade, "Ad-Hoc Multicast Routing Protocol", Internetdraft, Aug. 1998.

[7] C. Wu, Y. Tay, C. Toh, "Ad-Hoc Multicast Routing Protocol utilizing Increasing idnumbers (AMRIS): functional specification", Intemet-draft, November 1998.

[8] E.M. Royer and C.E. Perkins, "Multicast Ad-Hoc On-Demand Distance Vector (MAODV) Routing," Internet-Draft, draft-ietf-manet-maodv-00.txt, July 2000.

[9] Y. Yi, S. Lee, W. Su, and M. Gerla, "On-Demand Multicast Routing Protocol (ODMRP) for Ad-Hoc Networks," Internet-Draft, draft-yi-manet-odmrp-00.txt, March 2003.

[10]J. J. Garcia-Luna-Aceves and E.L. Madruga, "The Core-Assisted Mesh Protocol," IEEE JSAC, Aug. 1999, pp. 1380-94.

[11]Y. Ko, N. Vaidya: "Geocasting in Mobile Ad-Hoc Networks: Location-Based Multicast Algorithms", Technical Report TR-98-018, Texas A\&M University, September 1998.

[12]C. Jelger, T. Noel: "Unicast and Multicast Gatewaying in IPv6 Ad-Hoc networks", https://safari-rnrt.rd.francetelecom.com/Public/workshop_safari_2004/.

[13]W. Ding, "Multicast Routing in Fixed Infrastructure and Mobile Ad-Hoc Wireless Networks with a Multicast Gateway", Carleton University, Ontario, Canada, July 2002.

[14]P. Ruiz et al., "The MMARP Protocol for Efficient Support of Standard IP Multicast Communications in Mobile Ad-Hoc Access Networks". In proc. of the IST Mobile \& Wireless Comm. Summit 2003, June 2003.

[15]GloMoSim: A Scalable Simulation Environment for Wireless and Wired Network Systems. http://pcl.cs.ucla.edu/projects/glomosim/. 